Pacific Journal of Mathematics

COUNTABLE SPACES WITHOUT POINTS OF FIRST 


\title{
COUNTABLE SPACES WITHOUT POINTS OF FIRST COUNTABILITY
}

\author{
RONNIE LEVY
}

In this paper we show that there are $2^{c}$ non-homeomorphic countable regular spaces, each of which has no point of first countability. Several specific countable regular spaces are shown not to be homeomorphic.

1. Preliminaries. A countable space need not be first countable. One example of such a space is $N \cup\{p\}$ where $p \in \beta N-N$ and the topology is the relative topology of $\beta N$. This space, however, has many points of first countability-indeed all of the points of $N$ are isolated. Several examples of countable spaces without points of firs $\hat{\imath}$ countability are known to exist.

$N$ denotes the space of natural numbers including $0, Q$ denotes the space of rational numbers, and $\mathbf{R}$ denotes the space of reals. The cardinal of $\mathbf{R}$ is denoted $c$. If $X$ is a completely regular Hausdorff space, $\beta X$ is the Stone-Cech compactification of $X$. If $X$ and $Y$ are spaces and $f: X \rightarrow Y$ is a continuous surjection, $f$ is irreducible if there is no proper closed subset $K$ of $X$ such that $f(K)=Y$. It is well-known (see for example [11], 10.48) that if $X$ and $Y$ are compact Hausdorff spaces and $f: X \rightarrow Y$ is a continuous surjection, there is a closed subset $K$ of $X$ such that $f(K)=Y$ and the restriction of $f$ to $K$ is irreducible. A space $X$ is resolvable if $X$ contains disjoint dense subsets. A space $X$ is homogeneous if for any pair of points $p, q \in X$, there is a homeomorphism $f: X \rightarrow X$ such that $f(p)=q$. A rigid space is a space whose only auto-homeomorphism is the identity.

For $n \in N$, let $R_{n}$ be $N^{\{1, \cdots, n\}}$. Since for $n=0,\{1, \cdots, n\}=\varnothing$, $R_{0}=\{\varnothing\}$. The empty set, when viewed as the element of $R_{0}$, is denoted $p_{0}$. Let $S=\cup_{n \in N} R_{n}$ and define an order $\leqq$ on $S$ by $p \leqq q$ if and only if $p \in R_{m}, q \in R_{n}$ with $m \leqq n$ and $q \mid\{1, \cdots, m\}=p$. $(S, \leqq)$ is a tree (see [6]) and is clearly countably infinite. For $x \in S, A_{x}$ is the set $\{p \in S: x \leqq p, x \neq p$, and $x \leqq y \leqq p$ implies $x=y$ or $y=p\}$; thus, $A_{x}$ is the set of immediate successors of $x$. For $x \in S, U \subseteq N$, let $K_{x}^{U}=$ $\{x\} \cup\left\{p \in S\right.$ : There is a $q \in A_{x}$ such that the last entry of $q$ is an element of $U$ and $q \leqq p\}$.

If $p \in \beta N-N$, that is, $p$ is a free ultrafilter on $N$, then $\Sigma_{p}$ denotes the subspace $N \cup\{p\}$ of $\beta N$. Two points $p$ and $q$ of $\beta N-N$ are the same $\beta N$-type, or simply the same type, if $\Sigma_{p}$ is homeomorphic to $\Sigma_{q}$, or, 
equivalently, if there is a permutation $\varphi$ of $N$ such that the elements of $q$ are precisely the images under $\varphi$ of the elements of $p$.

All given spaces are assumed to be completely regular and Hausdorff.

For general background see [3] or [11].

2. Construction of $2^{c}$ countable spaces. Suppose $\mathcal{U} \in$ $\beta N-N$. A topology on $S$ is defined by taking as a subbase the collection $\left\{K_{x}^{U}: x \in S, U \in \mathcal{U}\right\}$. (It is not hard to show that in fact $\left\{K_{x}^{U}: x \in S, U \in \mathcal{U}\right\}$ is a base for a topology, although we do not need this fact.) The topological space which arises in this way is denoted $S_{\mathcal{U}}$. For each $U, S_{\mathcal{U}}$ is Hausdorff. To see this, notice first that if $n \in \in$, $N-\{n\} \in U$. Suppose $p=\left(p_{1}, \cdots, p_{m}\right)$ and $q=\left(q_{1}, \cdots, q_{n}\right)$ are distinct elements of $S$, with $m \leqq n$. If $p$ and $q$ are not comparable under $\leqq$, the sets $K_{p}^{N}$ and $K_{q}^{N}$ are disjoint neighborhoods of $p$ and $q$. If $p \leqq q$, $K_{p}^{N-\left\{p_{m+1}\right\}}$ and $K_{q}^{N}$ are disjoint neighborhoods of $p$ and $q$. Each $S_{\mathcal{U}}$ is also zero-dimensional. To check this, we show that each set of the form $K_{p}^{U}$ is closed, whether or not $U \in \mathcal{U}$. Suppose $q \notin K_{p}^{U}$. If $q$ and $p$ are not comparable, $K_{q}^{N}$ is a neighborhood of $q$ which does not intersect $K_{p}^{U}$. If $q \leqq p$, let $r=\left(r_{1}, \cdots, r_{k}\right)$ be the element of $A_{q}$ such that $r \leqq p$. Then $K_{q}^{N-\left\{r_{k}\right\}}$ is a neighborhood of $q$ which does not intersect $K_{p}^{U}$. If $p \leqq q$, then since $q \notin K_{p}^{U}$, it follows that $x \notin K_{p}^{U}$ for any $x$ such that $q \leqq x$, that is, $K_{q}^{N} \cap K_{p}^{U} \neq \varnothing$. This proves that $S_{U}$ is zero-dimensional. Furthermore, since a zero-dimensional Hausdorff space is regular, and since a regular Lindelof space is normal, $S_{q_{u}}$ is normal for each $\mathcal{U}$.

We now claim that for each $U, S_{\mathcal{U}}$ is homogeneous. It suffices to show that if $q \in S$, there is a homeomorphism $f_{q}: S_{U} \rightarrow S_{\mathscr{U}}$ such that $f_{q}(q)=p_{0}$, because then $f_{p}^{-1} \circ f_{q}$ is a homeomorphism of $S_{U}$ mapping $q$ to $p$. We first show that there is an order-preserving homeomorphism $g: S_{U} \rightarrow K_{q}^{N}$. Suppose $q=\left(n_{1}, \cdots, n_{k}\right)$. Define $g$ by $g\left(p_{0}\right)=q$, and $g\left(m_{1}, \cdots, m_{l}\right)=\left(n_{1}, \cdots, n_{k}, m_{1}, \cdots, m_{l}\right)$. The function $g$ is clearly oneto-one and onto. $g\left(K_{\left(m_{1}, \cdots, m_{l}\right)}^{U}\right)=K_{\left(n_{1}, \cdots, n_{k}, m_{1}, \cdots, m_{l}\right)}^{U}$ so $g$ is an open map. $g^{-1}\left(K_{\left(n_{1}, \cdots, n_{k}, m_{1}, \cdots, m_{l}\right)}^{U}\right)=K_{\left(m_{1}, \cdots, m_{l}\right)}^{U}$ so $g$ is continuous. Now define $f: S_{u} \rightarrow S_{u}$ by $f\left(K_{g(q)}^{N}\right.$ is the identity map, $f\left(S_{u}-K_{q}^{N}\right)=g\left(S_{u}-K_{q}^{N}\right)$, and $f \mid\left(K_{q}^{N}-K_{g(q)}^{N}\right)=g^{-1}\left(K_{q}^{N}-K_{g(q)}^{N}\right)$. Then $f \circ f$ is the identity map on $S_{u}$, so $f$ is one-to-one and onto. $f\left(p_{0}\right)=q$, so $f(q)=f\left(f\left(p_{0}\right)\right)=$ $p_{0}$. Furthermore, the restriction of $f$ to each of the clopen sets $\left(S_{\mathscr{U}}-K_{q}^{N}\right),\left(K_{q}^{N}-K_{g}^{N}(g)\right)$, and $K_{g(q)}^{N}$ is a homeomorphism, so $f$ is a homeomorphism.

$S_{\mathscr{U}}$ is not first countable. To see this, let the map $h: \Sigma_{\mathscr{U}} \rightarrow\left\{p_{0}\right\} \cup$ $A_{p_{0}}$ be defined by

$$
h(x)=\left\{\begin{array}{lll}
p_{0} & \text { if } & x=q \\
n & \text { if } & x=n
\end{array}\right.
$$


Then $h$ is a homeomorphism, so $p_{0}$ is not a point of first countability. Furthermore, since $S_{U}$ is homogeneous, $S_{u}$ has no point of first countability.

Finally, we observe that there are $2^{c}$ nonhomeomorphic spaces

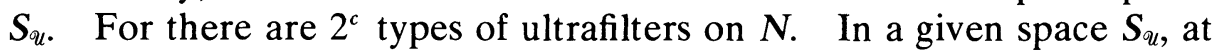
most $c$ spaces $\Sigma_{p}$ can appear as subspaces. Thus, if there were fewer than $2^{c}$ homeomorphism classes of $S_{u}$ 's, there would be fewer than $2^{c}$ types of ultrafilters, a contradiction.

We summarize in the following proposition.

Proposition 2.1. There are $2^{c}$ nonhomeomorphic countable regular homogeneous spaces each of which fails to have any point of first countability.

REMARK. The construction of $S_{u}$ can be modified to give rigid countable regular spaces without point of first countability. To do this, enumerate the elements of $S$ as $\left\{p_{k}: k \in N\right\}$ and let $\left\{\mathscr{U}_{k}: k \in N\right\}$ be a collection of free ultrafilters on $N$ such that if $k \neq n, \Sigma_{a_{k}}$ is not homeomorphic to any subspace of $\Sigma_{\mathscr{U}_{n}}$. Now take as a neighborhood base at $p_{k}$ the set $\left\{K_{p_{k}}^{U}: U \in \mathcal{U}_{k}\right\}$. Then $S$ with this topology can be shown to have the required properties. An argument similar to the counting argument used in proving 2.1 shows that there are $2^{c}$ such spaces no two of which are homeomorphic.

3. Other countable spaces. In this section we examine some other countable spaces without points of first countability. We will list for the sake of later reference some well-known and some easy facts.

Lemma 1. ([3], 9H). Every countable subset of an extremally disconnected space is $C^{*}$-embedded.

Lemma 2. ([8]). A space is extremally disconnected if and only if every dense subset is $C^{*}$-embedded.

LEMMA 3. A countable subspace of an extremally disconnected space is extremally disconnected.

Proof. If $A$ is a countable subspace of the extremally disconnected space $X$, every subset $A$ is $C^{*}$-embedded in $X$ (Lemma 1$)$ and hence in $A$. Therefore, by Lemma $2, A$ is extremally disconnected.

LEMMA 4. An extremally disconnected space without isolated points has no convergent sequence of distinct points and hence no point of first countability. 
Proof. If $a_{n} \rightarrow b$ where $a_{n} \neq a_{k}$, if $n \neq k$, the function $f:\left\{a_{n}\right\} \rightarrow \mathbf{R}$ defined by $f\left(a_{n}\right)=(-1)^{n}$ does not extend continuously to $b$, so the countable set $\left\{a_{n}\right\}$ is not $C^{*}$-embedded. Hence by Lemma 1 , no space which contains a nontrivial convergent sequence is extremally disconnected.

The following example was described in a letter by Professor W. Comfort. Let $\varphi: \beta N-N \rightarrow[0,1]$ be a continuous surjection. Let $K$ be a closed subset of $\beta N-N$ such that $\varphi(K)=[0,1]$ and $\varphi \mid K$ is irreducible. Let $A$ be a countable dense subset of $[0,1]$ and for each $a \in A$ choose $c_{a} \in(\varphi \mid K)^{-1}(a)$. Then $\left\{c_{a}: a \in A\right\}$ is a countable subset of $\beta N-N$ whose closure maps onto [0,1] by an irreducible map. Let $\mathscr{C}$ be the set of all countable subsets of $\beta N-N$ whose closures map onto $[0,1]$ by a continuous irreducible map. We have seen that $\mathscr{C} \neq \varnothing$. Now suppose $C \in \mathscr{C}$ and $f: C 1_{\beta N} C \rightarrow[0,1]$ is an irreducible map.

$C$ is resolvable. To see this, let $A_{1}$ and $A_{2}$ be disjoint dense subsets of $f(C)$. Then $f^{-1}\left(A_{1}\right) \cap C$ and $f^{-1}\left(A_{2}\right) \cap C$ are disjoint subsets of $C$. We claim that each is dense in $C$. $f\left(C 1_{\beta N}\left(f^{-1}\left(A_{1}\right) \cap C\right)\right)$ is a compact subset of $[0,1]$ containing the dense subset $A_{1}$ of $[0,1]$. Therefore, $f\left(C 1_{\beta N}\left(f^{-1}\left(A_{1}\right) \cap C\right)\right)=[0,1]$. Since $f$ is irreducible, $C 1_{\beta N}\left(f^{-1}\left(A_{1}\right) \cap C\right)=C 1_{\beta N} C$, so $f^{-1}\left(A_{1}\right) \cap C$ is dense in $C$. Similarly, $f^{-1}\left(A_{2}\right) \cap C$ is dense in $C$.

Since a resolvable space can have no isolated points, $C$ is dense-initself. By Lemma 3, the space $C$ is extremally disconnected. Lemma 4 implies that $C$ has no points of first countability, and, in fact, no nontrivial convergent sequences.

Every dense subset of $C 1_{\beta N} C$ is separable. The proof of this fact consists of observing that in the above argument that $f^{-1}\left(A_{1}\right) \cap C$ is dense in $C$, the crucial facts are that $C$ is dense in $C 1_{\beta N} C$ and that $f^{-1}\left(A_{1}\right) \cap C$ maps via $f$ onto a dense subset of $[0,1]$. Thus if $X$ is any dense subset of $C 1_{\beta N} C$ and $Y$ is any countable subset of $X$ such that $f(Y)$ is dense in $f(X)$, then $Y$ is dense in $X$. This shows that every dense subset of $C 1_{\beta N} C$ contains a dense subset which is in $\mathscr{C}$.

We summarize the above properties of elements of $\mathscr{C}$ in the following proposition.

Proposition 3.1. If $\mathscr{C}$ is the set of all countable subsets of $\beta N-N$ whose closures in $\beta N$ map onto $[0,1]$ by an irreducible map, then if $C \in \mathscr{C}$, $C$ is extremally disconnected, resolvable, and has no point of first countability. Furthermore, every dense subset of $C 1_{\beta N} C$ contains a dense subset which is itself an element of $\mathscr{C}$.

E. van Dowen, in [1], using a technique of Hewitt [5], shows that there are nonresolvable countable regular spaces without isolated points and with the property that any subset which has no isolated point and 
whose complement has no isolated point is open. Let $\mathscr{D}$ denote the collection of all such spaces. Then if $D \in \mathscr{D}$ and $U$ is an open subset of $D$, neither $C 1_{D} U$ nor $D-C 1_{D} U$ has isolated points. Hence, the closure of any open subset of $D$ is open, that is, $D$ is extremally disconnected. Therefore, by Lemma 4 , the space $D$ has no point of first countability.

Proposition 3.2. There are $2^{c}$ nonhomeomorphic extremally disconnected resolvable countable spaces without points of first countability and $2^{c}$ nonhomeomorphic extremally disconnected nonresolvable countable spaces without points of first countability.

Proof. Let $X$ be a countable extremally disconnected space without points of first countability (for example, $X \in \mathscr{C}$ or $X \in \mathscr{D}$ ). $\quad X$ is not countably compact - a countably compact countable space is compact and a compact extremally disconnected space has cardinal at least $2^{c}$. Therefore $X$ contains a closed copy of $N$, that is, $N$ may be viewed as a closed subspace of $X$. By Lemma 1 , the subspace $N$ is $C^{*}$. embedded in $X$, so $\beta N=C 1_{\beta X} N$ and $\beta N-N \subseteq \beta X-X$. Let $X_{0}=X$ and suppose a countable space $X_{\alpha}$ is defined for each $\alpha<\gamma$ where $\gamma$ is an ordinal less than $2^{c}$. Since a countable set has only $c$ subsets, at most $c|\gamma|=\max \{c,|\gamma|\}$ spaces $\Sigma_{p}$ are homeomorphic to a subset of some $X_{\alpha}$, $\alpha<\gamma$. Let $p_{\gamma} \in \beta N-N$ be an ultrafilter such that $\Sigma_{p_{\gamma}}$ is not homeomorphic to any subset of any $X_{\alpha}, \alpha<\gamma$. Let $X_{\gamma}=$ $X \cup\left\{p_{\gamma}\right\}$. Then $\left\{X_{\gamma}: 1 \leqq \gamma<2^{c}\right\}$ has cardinal $2^{c}$. If $\alpha<\gamma, X_{\gamma}$ contains the space $\Sigma_{p_{\gamma}}$ whereas $X_{\alpha}$ contains no subset homeomorphic to

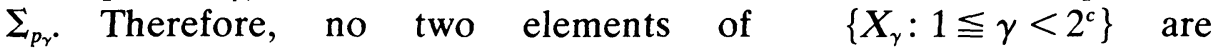
homeomorphic. For each $\gamma, X \subseteq X_{\gamma} \subseteq \beta X$, so each $X_{\alpha}$ is extremally disconnected and hence by Lemma 4 has no point of first countability. Since $X_{\alpha}-X$ consists of exactly one point for $\alpha \geqq 1$, each $X_{\alpha}$ is countable. Furthermore, since for a given $\alpha, X$ is dense and open in $X_{\alpha}$, every space $X_{\alpha}$ is resolvable if and only if $X$ is. Thus, if $X \in \mathscr{C}$, each $X_{\alpha}$ is resolvable and if $X \in \mathscr{D}$, each $X_{\alpha}$ is nonresolvable.

In [7] it is shown that every dense subset of $\beta Q-Q$ is separable. This fact is established by showing that there is a continuous surjection $F: \beta Q \rightarrow[0,1]$ such that $A$ is dense in $\beta Q$ if and only if $F(A)$ is dense in $[0,1]$. Let $\mathscr{B}$ be the set of countable dense subsets of $\beta Q-Q$. If $B \in \mathscr{B}$, then since $\beta Q-Q$ is dense in $\beta Q([3], 7 \mathrm{E}), B$ is dense in $\beta Q$ and therefore dense-in-itself. If $A_{1}$ and $A_{2}$ are disjoint dense subsets of $F(B)$, then by the above property of $F, F^{-1}\left(A_{1}\right) \cap B$ and $F^{-1}\left(A_{2}\right) \cap B$ are disjoint dense subsets of $B$. Therefore, $B$ is resolvable. Finally, since $B$ is dense in $\beta Q$, any point of first countability of $B$ would be a $G_{\delta}$ of $\beta Q([3], 9.7)$, and no point of $\beta Q-Q$ is a $G_{\delta}$ of $\beta Q([3], 9.6)$. Thus, $B$ has no point of first countability. 
Mrowka proves in [9] that any countable dense subset of $\mathbf{R}^{\mathbf{R}}$ with the product topology fails to have any point of first countability. Let $M$ be the set of countable dense subsets of $\mathbf{R}^{\mathbf{R}}$. Suppose $M \in \mathcal{M}$. Then $M$ is resolvable, since if $A_{1}$ and $A_{2}$ are disjoint dense subsets of $\{f(0): f \in M\}$, then $\left\{f \in M: f(0) \in A_{1}\right\}$ and $\left\{f \in M: f(0) \in A_{2}\right\}$ are disjoint dense subsets of $M$. It is proved in [7] that $M$ has no compactification each of whose dense subsets is separable.

Let $U$ be a free ultrafilter on $N$. For each $p_{k} \in A_{p_{0}}$ choose $x_{k} \in A_{P_{k}}$. Let $W=\bigcup_{k=1}^{\infty} K_{x_{k}}^{N}$. The subset $W$ is open in $S_{\mathscr{U}}$ and $C 1_{s_{u}} W=W \cup\left\{p_{0}\right\}$ which is not open in $S_{\vartheta}$. Thus $S_{u}$ is not extremally disconnected. $S_{u}$ can be written as the union of disjoint dense subsets by $S_{U}=\bigcup_{k=0}^{\infty} R_{2 k} \cup \bigcup_{k=0}^{\infty} R_{2 k+1}$. Therefore, $S_{\mathscr{U}}$ is resolvable.

We now combine the above comments.

PROPOSITION 3.3. Let $\mathscr{C}$ denote the set of all countable subsets of $\beta N-N$ whose closures map irreducibly onto $[0,1]$. Let $\mathscr{D}$ denote the class of countable, nonresolvable spaces without isolated points such that each set without isolated points and whose complement has no isolated points is open. Let $\mathscr{B}$ be the set of countable dense subsets of $\beta Q-Q$. Let $\mathcal{M}$ denote the set of countable dense subsets of $\mathbf{R}^{\mathbf{R}}$. Suppose $C \in \mathscr{C}, D \in \mathscr{D}$, $B \in \mathscr{B}, M \in \mathcal{M}$, and $\mathcal{U} \in \beta N-N$.
(i) $M$ is not homeomorphic to $B, C$, or $D$.
(ii) $D$ is not homeomorphic to $B, C$, or $S_{\vartheta}$.
(iii) $C$ is not homeomorphic to $S_{थ \text {. }}$.

Proof. (i) $C$ and $D$ are extremally disconnected whereas $M$ is not. $B$ has a compactification every dense subset of which is separable whereas $M$ has no such compactification.

(ii) $D$ is not resolvable whereas each of the spaces, $B, C$, and $S_{\mathscr{U}}$ is resolvable.

(iii) $C$ is extremally disconnected whereas $S_{\mathcal{U}}$ is not.

We can summarize Proposition 3.3 in the following graph, the edges of which represent nonhomeomorphism:

$(*)$

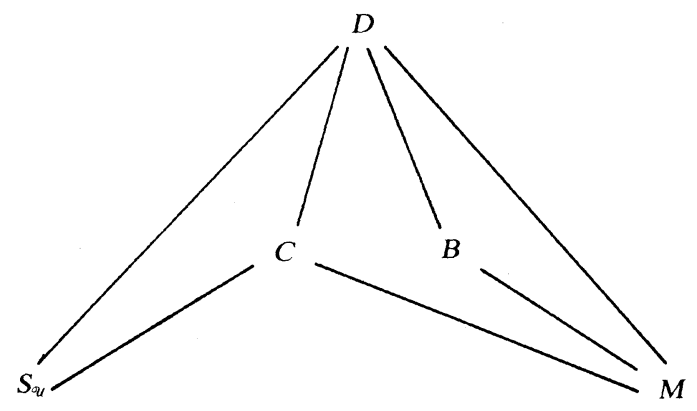


4. Completing the graph. The graph $(*)$ is not complete because no edge joins $B$ and $C$, or $B$ and $S_{\varkappa}$, or $M$ and $S_{\varkappa}$. In this section we show that if $U, B$, and $M$ are chosen in particular ways, the graph can be completed. We also show that there are elements of $\mathscr{C}$ and $\mathscr{B}$ which are homeomorphic. We need several facts.

Theorem (Efimov, [2]). Any extremally disconnected space of weight at most $c$ (in particular, any countable extremally disconnected space) can be embedded in $\beta N$.

A point $p \in \beta Q-Q$ is called a remote point of $\beta Q$ if $p$ is not in the closure of any discrete subset of $Q$. Eric van Dowen has recently proved the following.

THEOREM (van Dowen). $\beta Q$ has a dense set of remote points.

THEOREM (Woods, [12]). Suppose $X$ is a dense subspace of $\beta Q$. Then $X$ is extremally disconnected if and only if every point of $X$ is a remote point of $\beta Q$.

Proposition 4.1. There are homeomorphic spaces $B$ and $C$ such that $B \in \mathscr{B}$ and $C \in \mathscr{C}$. In fact, every extremally disconnected element of $\mathscr{B}$ is homeomorphic to an element of $\mathscr{C}$.

Proof. We first note that there are extremally disconnected elements of $\mathscr{B}$. For by van Dowen's theorem, the set of remote points of $\beta Q$ is dense in $\beta Q-Q$, and so by Wood's theorem, any countable dense subset of the set of remote points is extremally disconnected. By Efimov's theorem, $B$ is homeomorphic to a subspace $C$ of $\beta N-N$. We show $C \in \mathscr{C}$. The countable space $C$ is $C^{*}$-embedded in $\beta N$ and hence in $C 1_{\beta N} C$, that is, $C 1_{\beta N} C=\beta C$. Let $f: C \rightarrow B$ be a homeomorphism and $f^{*}: \beta C \rightarrow \beta Q$ be its Stone extension. Let $g: Q \rightarrow Q \cap[0,1]$ be a homeomorphism and let $g^{*}: \beta Q \rightarrow[0,1]$ be its Stone extension. $f^{*}$ and $g^{*}$ are each irreducible $([3], 6.12)$. It follows that $g^{*} \circ f^{*}: C 1_{\beta N} C \rightarrow[0,1]$ is irreducible. Therefore, $C \in \mathscr{C}$.

Remarks. 1. By Efimov's theorem, every countable extremally disconnected space without points of first countability is embeddable in $\beta N-N$. However, not every such space is homeomorphic to an element of $\mathscr{C}$. For example, by 3.3 no element of $\mathscr{D}$ is homeomorphic to an element of $\mathscr{C}$.

2. We do not know if every element of $\mathscr{C}$ is homeomorphic to an element of $\mathscr{B}$.

Proposition 4.1 says that some elements of $\mathscr{B}$ are homeomorphic to 
elements of $\mathscr{C}$. However, if $B$ is any countable dense subset of $\beta Q-Q$ which contains a nonremote point of $\beta Q$, then by Wood's theorem, $B$ is not extremally disconnected and hence not homeomorphic to an element of $\mathscr{C}$.

PROPOSITION 4.2. There are elements of $\mathscr{B}$ which are not homeomorphic to any elements of $\mathscr{C}$.

We do not know if there is an ultrafilter $U \in \beta N-N$ and a space $M \in M$ such that $S_{u}$ is homeomorphic to $M$. There are, however, elements of $\mathcal{M}$ which are not homeomorphic to $S_{u}$ for any $\mathcal{U} \in \beta N$ $N$. For example, if $M$ is the set of polynomials with rational coefficients, $M$ has convergent sequences of distinct elements, such as the sequence of constant functions $\{1 / n: n \in N\}$. It can be shown that none of the spaces $S_{u}$ has any nontrivial convergent sequence.

We also do not know if any of the spaces $S_{U}$ is homeomorphic to any element of $\mathscr{B}$. However, by 2.1 , for a given $B \in \mathscr{B}$ and a given $M \in \mathcal{M}$, there is a $U \in \beta N-N$ such that $S_{u}$ is homeomorphic to neither $B$ nor $M$.

Proposition 4.3. Suppose $\mathscr{C}, \mathscr{D}, \mathscr{B}$, and $\mathcal{M}$ are the collections of spaces defined in Proposition 3.3. Then there is a $B_{0} \in \mathscr{B}, M_{0} \in \mathcal{M}$, and $\mathcal{U}_{0} \in \beta N-N$ such that if $C \in \mathscr{C}$ and $D \in \mathscr{D}$, no two of the spaces $B_{0}, C, D$, $M_{0}, S_{\mathcal{U}_{0}}$ are homeomorphic.

Proof. Choose $B_{0}$ as in 4.2 , let $M_{0}$ be any element of $\mathcal{M}$, and choose $u_{0} \in \beta N-N$ such that $S_{\mathcal{U}_{0}}$ is homeomorphic to neither $B_{0}$ nor $M_{0}$.

Proposition 4.3 can be summarized in the following complete graph, where again the edges represent nonhomeomorphism.

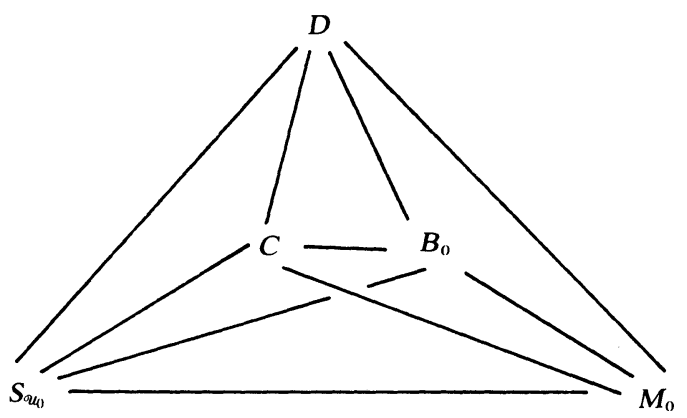

5. A final observation. A famous theorem of of Sierpinski characterizes the rationals among metric spaces. Since a countable first 
countable space is metrizable by the Urysohn metrization theorem, Sierpinski's theorem can be stated as follows.

THEOREM (Sierpinski, [10]). Any countable first countable space without isolated points is homeomorphic to $Q$.

By 2.1 (or 3.2 or 3.3 or 4.3 ), there is no such characterization for countable spaces without points of first countability. However, we note that all such spaces share the property that they have weaker topologies homeomorphic to $Q$.

Proposition 5.1. If $X$ is countable and has no isolated point, then there is a continuous, one-to-one function from $X$ onto $Q$.

Proof. For each $p, q \in X, p \neq q$, let $U_{p, q}$ be a clopen subset of $X$ containing $p$ but not $q$. The collection $\left\{U_{p, q}: p, q \in X, p \neq q\right\} \cup\{X-$ $\left.U_{p, q}: p, q \in X, p \neq q\right\}$ is a countable subbase for a (regular) topology on set $X$. Let $\hat{X}$ denote the set $X$ with this new topology. $\hat{X}$ is second countable and has no isolated point (since $X$ has no isolated point) and hence, by Sierpinski's theorem, is homeomorphic to $Q$.' Thus, $X$ has a weaker topology homeomorphic to $Q$.

\section{REFERENCES}

1. E. van Dowen, Simultaneous extension of continuous functions, Free University, Thesis.

2. B.A. Efimov, Extremally disconnected compact spaces and absolutes, Trans. Moscow Math. Soc. 23 (1970), 243-285.

3. L. Gillman and M. Jerison, Rings of continuous functions, Van Nostrand (1960).

4. P. W. Harley, III, A countable nowhere first countable Hausdorff space, Canad. Math. Bull. 16 (1973), 441-442.

5. E. Hewitt, A problem of set-theoretic topology, Duke Math. J. 10 (1943), 309-333.

6. R. Levy, Showering spaces, Pacific J. Math. 57 (1975), 223-232.

7. R. Levy and R. H. McDowell, Dense subsets of $\beta X$, Proc. Amer. Math. Soc. 50 (1975), 426-430.

8. R.H. HcDowell, Extension of functions from dense subspaces, Duke Math. J., 25 (1958), 297-304.

9. S. Mrowka, On the potency of compact spaces and the first axiom of countability, Bull. Acad. Polon. Sci., Ser. Math. Ast. Phys., 6 (1958), 7-9.

10. W. Sierpinski, Sur une propriete topologique des ensembles denombrables denses en soi, Fund. Math., 1 (1920), 11-16.

11. R. Walker, The Stone-Cech compactification, Springer (1974).

12. R. G. Woods, Co-absolutes of remainders of Stone-Cech compactifications, Pacific J. Math., 37 (1971), 545-560.

Received October 25, 1976 and in revised form March 23, 1977. The author wishes to thank the referee for many helpful suggestions.

George Mason University

FAIRFAX, VA 22030 



\section{PACIFIC JOURNAL OF MATHEMATICS \\ EDITORS}

RICHARD ARENS (Managing Editor)

University of California

Los Angeles, CA 90024

\section{R. A. Beaumont \\ University of Washington \\ Seattle, WA 98105}

C. C. MOORE

University of California

Berkeley, CA 94720

\section{J. DUGUNDJI}

Department of Mathematics

University of Southern California

Los Angeles, CA 90007

R. FINN AND J. MILGRAM

Stanford University

Stanford, CA 94305

\section{ASSOCIATE EDITORS}

\section{E. F. BECKENBACH}

B. H. NeUmanN

F. WOLF

K. YoSHIDA

\section{SUPPORTING INSTITUTIONS}

\author{
UNIVERSITY OF BRITISH COLUMBIA \\ CALIFORNIA INSTITUTE OF TECHNOLOGY \\ UNIVERSITY OF CALIFORNIA \\ MONTANA STATE UNIVERSITY \\ UNIVERSITY OF NEVADA \\ NEW MEXICO STATE UNIVERSITY \\ OREGON STATE UNIVERSITY \\ UNIVERSITY OF OREGON \\ OSAKA UNIVERSITY
}

\author{
UNIVERSITY OF SOUTHERN CALIFORNIA \\ STANFORD UNIVERSITY \\ UNIVERSITY OF HAWAII \\ UNIVERSITY OF TOKYO \\ UNIVERSITY OF UTAH \\ WASHINGTON STATE UNIVERSITY \\ UNIVERSITY OF WASHINGTON \\ AMERICAN MATHEMATICAL SOCIETY
}

The Supporting Institutions listed above contribute to the cost of publication of this Journal, but they are not owners or publishers and have no responsibility for its contents or policies.

Mathematical papers intended for publication in the Pacific Journal of Mathematics should be in typed form or offset-reproduced (not dittoed), double spaced with large margins. Underline Greek letters in red, German in green, and script in blue. The first paragraph or two must be capable of being used separately as a synopsis of the entire paper. Items of the bibliography should not be cited there unless absolutely necessary, in which case they must be identified by author and Journal, rather than by item number. Manuscripts, in duplicate, may be sent to any one of the four editors. Please classify according to the scheme of Math. Reviews, Index to Vol. 39. All other communications should be addressed to the managing editor, or Elaine Barth, University of California, Los Angeles, California, 90024.

100 reprints are provided free for each article, only if page charges have been substantially paid. Additional copies may be obtained at cost in multiples of 50 .

The Pacific Journal of Mathematics is issued monthly as of January 1966. Regular subscription rate: $\$ 72.00$ a year (6 Vols., 12 issues). Special rate: $\$ 36.00$ a year to individual members of supporting institutions.

Subscriptions, orders for back numbers, and changes of address should be sent to Pacific Journal of Mathematics, 103 Highland Boulevard, Berkeley, California, 94708.

PUBLISHED BY PACIFIC JOURNAL OF MATHEMATICS, A NON-PROFIT CORPORATION

Printed at Jerusalem Academic Press, POB 2390, Jerusalem, Israel.

Copyright (C) 1977 Pacific Journal of Mathematics All Rights Reserved 


\section{Pacific Journal of Mathematics \\ Vol. 70, No. $2 \quad$ October, 1977}

B. Arazi, A generalization of the Chinese remainder theorem ........... 289

Thomas E. Armstrong, Polyhedrality of infinite dimensional cubes .... . . . 297

Yoav Benyamini, Mary Ellen Rudin and Michael L. Wage, Continuous

images of weakly compact subsets of Banach spaces ............ 309

John Thomas Burns, Curvature functions on Lorentz 2-manifolds ......... 325

Dennis F. De Riggi and Nelson Groh Markley, Shear distality and equicontinuity .................................. 337

Claes Fernström, Rational approximation and the growth of analytic

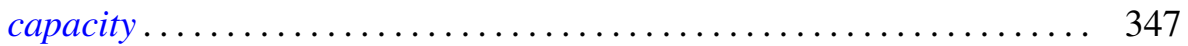

Pál Fischer, On some new generalizations of Shannon's inequality....... 351

Che-Kao Fong, Quasi-affine transforms of subnormal operators ......... 361

Stanley P. Gudder and W. Scruggs, Unbounded representations of

*-algebras........................................ 369

Chen F. King, A note on Drazin inverses .................... 383

Ronald Fred Levy, Countable spaces without points of first countability . . . 391

Eva Lowen-Colebunders, Completeness properties for convergence

spaces ......................................... 401

Calvin Cooper Moore, Square integrable primary representations ....... 413

Stanisław G. Mrówka and Jung-Hsien Tsai, On preservation of

E-compactness ................................ 429

Yoshiomi Nakagami, Essential spectrum $\Gamma(\beta)$ of a dual action on a von

Neumann algebra ................................ 437

L. Alayne Parson, Normal congruence subgroups of the Hecke groups

$G\left(2^{(1 / 2)}\right)$ and $G\left(3^{(1 / 2)}\right)$...

Louis Jackson Ratliff, Jr., On the prime divisors of zero in form rings . . . . 489

Caroline Series, Ergodic actions of product groups .................. 519

Robert O. Stanton, Infinite decomposition bases..................... 549

David A. Stegenga, Sums of invariant subspaces .................. 567 